\title{
Distúrbios relacionados ao álcool em um setor de urgências psiquiátricas. Ribeirão Preto, Brasil (1988-1990) 1
}

\author{
Alcohol-related disorders in a psychiatric emergency \\ service. Ribeirão Preto, Brazil (1988-1990) 1
}

Eliene Reis de Oliveira 2

Margarita A. Villar Luis 3

\footnotetext{
1 Subvencionado pelo Consel ho Nacional de Desenvolvimento Científico e Tecnológico - CNPq.

2 Escola de Enfermagem de Ribeirão Preto,

Universidade de São Paulo.

Ribei rão Preto, SP,

14040-902, Brasil.

3 Departamento de

Enfermagem Psiquiátrica e

Ciências Humanas,

Escola de Enfermagem de

Ribeirão Preto,

Universidade de São Paulo.

Ri bei rão Preto, SP,

14040-902, Brasil.
}

Abstract This study reports the results of a survey carried out in the psychiatric emergency service of the University Hospital of Ribei rão Preto, Brazil (1988-1990) with the purpose of characterizing psychiatric disorders related to the consumption of alcohol. The sample included data on a total of 1,082 patients diagnosed during this period. A total of 576 cases were diagnosed with "alcohol dependency syndrome", 379 with "alcohol psychosis", and 127 patients with "al cohol without dependency". The majority of patients were male and the age group most affected was 25-44 years for both sexes. The study calls attention for the importance of alcohol abuse as a public health problem.

Key words Mental Health; Psychiatry; Alcoholism; Public Health

Resumo Este estudo apresenta o resul tado de uma investi gação conduzida no setor de urgências psi quiátricas do Hospital das Clíni cas de Ribei rão Preto, Universidade de São Paulo (19881990), objetivando caracterizar distúrbi os pasiquiátricos relaci onados ao consumo de ál cool. A amostra incluiu um total de 1.082 pacientes diagnosticados durante o período da investigação. Ao todo, 576 casos foram di agnosticados com "síndrome de dependência al coólica", 379 com "psicose al coóli ca" e 127 paci entes com “ál cool sem dependência”. A mai oria dos atendi mentos recaiu sobre pacientes do sexo mascul ino e o grupo de idade mai s afetado foi , para ambos os sexos, o de 25-44 anos. O estudo chama atenção para a importância do abuso de álcool como um problema em saúde pública.

Palavras-chave Saúde Mental; Psiquiatria; Alcoolismo; Saúde Pública 


\section{Introdução}

No decorrer de nossa formação profissional, durante as atividades de aprendizado nos serviços de saúde, pudemos constatar a freqüência com que apareciam os problemas relacionados ao uso de bebidas alcoólicas. Trata-se de relatos de familiares de pessoas que faziam uso abusivo de álcool, ou até mesmo do próprio consumidor dessa substância. Tal fato despertou nosso interesse em conhecer mais detalhadamente o problema do consumo de álcool.

O consumo deálcool parece ser o hábito social mais antigo e disseminado entre as populações, pois ele está associado a ritos religiosos e lhe é atribuída uma variedade de efeitos, tais como calmante, afrodisíaco, estimulante do apetite, desinibidor e outros. Seu uso vem desde a pré-história. Porém, somente neste sécuIo, foram realizados estudos mais sistematizados, voltando-se para os problemas que o consumo de álcool vem ocasionando às populações (Cardin et al., 1986).

O álcool pode ser proveniente de plantas de fácil cultivo como cevada, uvas, grãos de cereais e outros, que passarão por processos de fermentação e destilação, tornando-se a droga psicoativa mais consumida de todos tempos, como, por exemplo, o vinho ou similar, que desde a antigüidade é indispensável a comemorações, em momentos de al egria, ou como companheiro das desilusões (Masur, 1977).

Nas bebidas utilizadas pelo homem, está contido o álcool etanol ou álcool etílico que foi descrito pela primeira vez em 1300, na Idade M édia, tendo sua síntese ocorrido em 1854. Pertence ao subgrupo das funções oxigenadas denominado álcoois, que, por definição, são compostos orgânicos que possuem uma ou mais hodroxidrilas $(\mathrm{OH})$ ligadas diretamente ao átomo de carbono não-pertencentes a um núcleo benzênico (Reginato, 1986).

O etanol é uma substância psicoativa com capacidade de produzir alterações no funcionamento do sistema nervoso central (SNC), podendo modificar o comportamento dos indivíduos que dela fazem uso (MS, 1990).

Ao longo dos anos, o consumo de álcool vem se constituindo num grave problema médico-social, passando a desafiar os trabalhadores da saúde na sua capacidade de atuação objetiva. No Brasil, atualmente há uma demanda por parte das empresas, solicitando profissionais qualificados para tratamento específico de alcoolismo entre seus funcionários; existe também, por sua vez, uma demanda da população, que também requer esses profissionais, só que sob a forma de necessidade sentida, a qual não é menos explícita que as das empresas (Cardin et al., 1986).

\section{Conceituações sobre o alcoolismo}

O diagnóstico de alcoolismo tem sido definido com base em quatro grupos de indicadores (Schuckit, 1985): consumo, dependência psicológica, dependência física e problemas relacionados ao álcool. Hill (1985) apud Edwards (1985) dizia, em meados da década de 80, não existirem evidências do alcoolismo ser ou não uma doença. A ausência de um padrão homogêneo, de um curso único, ao contrário do que se pensava antes, faz com que al guns autores prefiram ver o alcoolismo como uma síndrome e não como uma doença.

O alcoolismo, que vem a ser um grande problema social, é visto como uma toxicomania pela OMS (1970), que a conceitua como "um estado psíquico e al gumas vezes também físico, resultante da interação entre um organismo vivo e uma substância, caracterizado por um comportamento e outras reações que incluem sempre compulsão para ingerir a droga, de forma contínua ou periódica, com a finalidade de experimentar seus efeitos psíquicos e às vezes para evitar o desconforto de sua abstinência. A tolerância pode existir ou faltar e o indivíduo pode ser dependente de mais de uma droga". Essa conceituação refere-se à toxicomania ou farmacodependência; nesse caso o alcoolismo fica caracterizado, por se verificar que o alcoólatra tende a aumentar progressivamente as doses ingeridas e, quando interrompe completa ou bruscamente a ingestão de álcool, apresenta o grave conjunto de sinais e sintomas físico-psíquicos que caracterizam a Síndrome de Abstinência Alcoólica.

A seguir, a OMS apresentou definições de alcoolismo e de alcoólatra:

"Al coolismo é uma doença de natureza complexa, na qual o ál cool atua como fator determinante sobre causas psi cossomáticas preexistentes no indivíduo e para cujo tratamento é preciso recorrer a processos profiláticos e terapêuticos degrande amplitude".

"Alcoól atras são bebedores excessi vos, cuja dependência do álcool chega a ponto de acarretar-Ihes perturbações mentais evi dentes, manifestações que afetam a saúde física e mental, suas reações individuais, seu comportamento sócio-econômi co ou pródomos de perturbações desse gênero eque, por isso, necessitam de tratamento".

$\mathrm{Na}$ atualidade, houve substituição desse termo, passando-se a denominar os usuários 
“bebedores-problema" e os dependentes de álcool "alcoolistas" (MS, 1990).

O termo alcoolismo foi empregado pela primeira vez em 1856 pelo médico sueco MagnusHuss, para designar os sinais e sintomas físicopsíquicos surgidos pela excessiva ingestão de doses elevadas (e por tempo prolongado) de bebidas alcoólicas (Fortes, 1975).

Jellinek (1946) apud Fortes (1975), um dos mais brilhantes pesquisadores do "Yale Center of Alcohol Studies", define que o alcoolismo é qualquer uso de bebidas alcoólicas que ocasiona prejuízos ao indivíduo, à sociedade ou a ambos.

Masur (1984) tem a convicção de que o alcoolismo é uma doença, na medida em que implica uma situação de dependência tão intensa que leva a visível prejuízo físico e/ ou interpessoal e correlaciona a sua existência à perda da liberdade sobre o ato de beber.

Em relação às causas do alcoolismo, há controvérsias. Há autores que admitem os sintomas da intoxicação crônica pelo álcool como sendo roupagens que escondem ou disfarçam uma personalidade mórbida. Não haveria propriamente alcoolismo primário, sendo os casos relatados como al coolismo secundários a "algo" subjacente à personalidade do paciente. Em outras palavras, o indivíduo normal nunca se tornaria um alcoolista crônico. Haveria sempre necessidade da existência de um fator ligado a uma personalidade mórbida (Fortes, 1975).

Pacheco \& Amaral (1944) apud Fortes (1975), no departamento de Neuropsiquiatria da Associação Paulista de Medicina, analisando internações motivadas por alcoolismo, assinalaram o importante contingente representado pelo alcoolismo secundário. Contrário a esse ponto de vista, Mayer-Gross (1958) apud Fortes (1975) relata que nem todos os alcoólatras teriam personalidade fortemente predisposta e argumenta que a massa de alcoolistas não provém unicamente deste ou daquele grupo em particular, constituindo sempre um grupo muito heterogêneo.

Sonnenreich (1971) defende categoricamente ser o alcoolismo uma doença adquirida à revelia de quaisquer predisposições psicossomáticas preexistentes: é decorrente do uso contínuo do próprio álcool.

Masur (1984), no concernente à causa, refere não haver uma resposta breve, clara e convincente; existem várias tentativas de respostas, como a vulnerabilidade biológica, a psicológica e a social. Isto partindo da premissa de que todos os que bebem têm potencialmente a possibilidade de se tornar alcoolistas. A maior ou menor probabilidade vai depender da interação entre os diferentes fatores da vulnerabilidade.

A título de conclusão, parece não haver uma causa única ou isolada, o ato de beber pode resultar de uma variedade de inter-relações de influências. Cada variante repercute de forma ímpar em cada pessoa (Cervesato, 1993).

Aspectos sócio-econômicos

relacionados ao consumo de álcool

Fortes (1975) apud Cervesato (1993) relata que o alcoolismo, pelas complicações sobrevindas no plano somático e na esfera psíquica da pessoa, e pela profunda repercussão no meio social, figura hoje como um dos mais graves problemas de saúde no Brasil.

Segundo Bertolote (1979), o al coolismo alcançou, hoje em dia, proporções cada vez mais crescentes, que podem ser estimadas por indicadores do nível de produção e consumo per capita, e pela freqüência de seu diagnóstico tanto no Brasil quanto em outros países.

O mesmo autor ressaltou que a produção nacional anual absoluta de álcool atingiu, em 1970, a média de 4,7 litros para cada brasileiro com 15 anos e mais idade. Walsh \& Grant (1985) observaram que, em todo o mundo, a produção de álcool cresceu em 50\% de 1965 a 1980, e a produção per capita em 15\% no mesmo período. Esses autores também verificaram que o consumo de álcool per capita, nos países em desenvolvimento, está crescendo num ritmo mais acentuado em relação aos países desenvolvidos, principalmente nos países africanos e latino-americanos, enquanto que na França e em Portugal o consumo per capita de álcool está diminuindo.

Masur \& Jorge (1986), em acréscimo a essas informações, têm a ressaltar que a indústria de bebidas alcoólicas ocupa a terceira posição na geração de impostos, sendo responsável por $10 \%$ de todo o imposto sobre produtos industriais (IPI). Fato esse que comprova os interesses econômicos que estão subjacentes através dos veículos de propaganda.

Assim, fatores como o mundo da oferta de bebidas alcoólicas, a desorganização social e o aumento do desemprego preocupam os profissionais da saúde, com o temor de que a magnitude dos problemas relacionados ao álcool e alcoolismo venha a aumentar e acabe por neutralizar as escassas tentativas de prevenção (Naveillan \& Vargas, 1984).

Para Cardin et al. (1986), o fato de o alcoolismo estar atingindo, no Brasil, faixas da população economicamente ativa, consolida essa 
patologia como um problema de saúde pública, entretanto, apesar dessa constatação, os autores ressalvam a escassez de estudos recentes efetuados no seio da população.

Os dados sobre a prevalência de problemas relacionados ao consumo de ál cool são variáveis. Robins et al. (1984) estimam que pode ser até $16 \%$ para uma população ao longo da vida. Os principais prejuízos decorrentes dessa situação se referem a acidentes de trânsito, diminuição da atividade produtiva, dificuldades de relacionamento interpessoal e doenças orgânicas conseqüentes ao consumo exagerado e crônico de álcool (Vaillant, 1983).

Nos Estados Unidos, o alcoolismo e o abuso de álcool constituem o principal problema de saúde em termos de custos econômicos, pois estima-se que 25 a $40 \%$ dos leitos de hospitais gerais estejam ocupados por pacientes vítimas das complicações do alcoolismo (Holden, 1987). Estudo recente concluiu que, em $1987,4,9 \%$ das mortes por todas as causas nesse país eram atribuíveis ao álcool, sendo o consumo de álcool responsável naquele ano pela perda de mais de 2,7 milhões de anos potenciais de vida (years of potential lifelost).

No Brasil, o problema também atinge grandes proporções, em função da existência de dados comprobatórios do mesmo, uma vez que, o álcool está relacionado com 1,5 milhões de acidentes de trânsito por ano (Masur \& Del Porto, 1982) e com 39\% de todas as ocorrências policiais, em igual período de tempo (SSPESP, 1980). Outra conseqüência refere-se ao afastamento dos trabalhadores por auxílio-doença, sendo que, no Brasil, em 1975, foi a terceira causa de afastamento por diagnóstico psiquiátrico, e como manutenção do auxílio-doença ocupou o quarto lugar (Cabernite (1982); Freitas (1986) apud Cardin et al., 1986). Fridman \& Pellegrini (1994), em dados mais recentes, confirmam a importância do problema evidenciando o alcoolismo como terceiro motivo de absenteísmo no trabalho e oitava causa de concessão de auxílio-doença pela Previdência Social.

O Detran de Porto Alegre apurou que, em metade dos acidentes de trânsito, havia pelo menos uma pessoa alcoolizada. Ao considerar apenas os acidentes com vítimas fatais, esta proporção subiu para 75\%. Em São Paulo, o estudo dos prontuários de 1.136 acidentes de trânsito com vítimas, entre 1976 e 1985, revelou que $18 \%$ envolviam o uso abusivo de bebidas alcoólicas. Apesar da magnitude, acreditase que estes números podem estar subestimados (MS, 1988).
Aspectos de saúde pública relacionados ao consumo de álcool

Dentre a população que consome bebidas alcoólicas, aproximadamente $10 \%$ desenvolvem o quadro de dependência do álcool (alcoolismo), que se caracteriza por alterações decorrentes do consumo excessivo, a nível social, psicológico e orgânico (American Psychiatric Association, 1987).

Raeder \& Cotrim (1990), num levantamento postal efetuado por dependências de drogas (CID 9 - 291) e quadros relacionados ao alcoolismo (CID 9 - 303, 304), incluíram 436 hospitais e clínicas psiquiátricas do Brasil, dos quais somente $36,5 \%$ contribuíram para avaliar a magnitude do problema de dependência e psicoses por álcool entre a população brasileira.

Os resultados dessa pesquisa indicaram que o número de internações envolvendo consumo de álcool é bastante preocupante - mais de 60 mil pessoas por ano. Cabe ressaltar que essas internações atingiram especialmente pessoas do sexo masculino com mais de 30 anos, ou seja, uma camada da população que constitui, hoje, o principal contingente da força de trabalho brasileiro.

As patologias associadas ao álcool estão entre os mais sérios problemas psiquiátricos enfrentados. Masur \& Jorge (1986) relatam que o próprio Ministério da Saúde informava, em 1981, que os diagnósticos relacionados ao álcool constituíam o segundo grupo mais freqüente entre todas as internaçõs psiquiátricas dos últimos anos. Dados recentes da Secretaria da Saúde mostram que cerca de $50 \%$ das internações psiquiátricas do sexo masculino são por alcoolismo (Laranjeira, 1995).

No Estado de São Paulo, não há um padrão de consumo de álcool e drogas entre a população, embora tenham sido realizados alguns estudos de prevalência de abuso de álcool e alcoolismo, os quais utilizaram vários tipos de amostragem da população geral, bem como questionários e critérios diagnósticos diferentes. Apesar disso, os resultados têm apresentado concordância em dados, tais como: “É maior o consumo de álcool na população masculina e o diagnóstico de abuso de álcool seria cerca de 12-15\% neste grupo; a maior parte dessas pessoas não busca tratamento; as muIheres bebem menos, com prevalência de diagnóstico de abuso em torno de 3-5\%, com alguma evidência mostrando aumento deste número". Contudo esses, os dados são muito parecidos com os da literatura internacional, tendendo a apresentar uma prevalência ligeiramente superior (Laranjeira, 1995). 
É digno de nota o trabalho efetuado por Azoubel Neto (1965) na cidade de Ribeirão Preto, onde constatou uma porcentagem de $13 \%$ de primeiras internações por alcoolismo (18.410 casos) no período de 1960 a 1962. Além disso, o autor verificou, para o ano de 1962, uma prevalência de $17 \%$ e $10 \%$ para o sexo masculino e feminino respectivamente, na categoria bebedor excessivo, sendo que, para o bebedor patológico, apareceu apenas o sexo masculino com uma prevalência de 13,6\%. Apesar da relevância desse estudo, não têm sido efetuados outros trabalhos dessa natureza, abordando o alcoolismo na região.

Aspectos relacionados ao indivíduo que abusa do álcool (Alcoolismo)

Poikolainen (1987), em um estudo com alcoolistas em tratamento de abstinência, mostrou que o consumo de álcool referido pelos pacientes variava em mais de duas vezes, segundo o tipo de instrumento utilizado para mensurá-lo. Por outro lado, a estimativa do consumo referido não foi homogênea. De modo geral, os bebedores excessivos são aquel es que mais tendem a minimizar o volume de bebida ingerido.

A esse respeito, há concordância com Masur et al. (1985), que questionam o quanto a história obtida sobre os hábitos de ingestão de bebidas alcoólicas de um paciente deve ser valorizada no direcionamento de hipóteses diagnósticas, já que é uma pergunta comumente formulada em ambientes clínicos, tanto ambulatoriais como hospitalares, onde, geralmente, é respondida como..."às vezes, mas só um pouco" ..."uma ou duas doses por dia" ..."bebo, mas não sou viciado" ..."só tomo álcool como aperitivo". Para essa autora, tais respostas geram muitas dúvidas por serem vagas e imprecisas, principalmente, quanto à veracidade, visto a tendência do usuário a negar o consumo abusivo de álcool para não ser rotulado como alcoólatra, dada a conotação moralista subjacente; esse é o principal fator que faz com que os pacientes minimizem o seu consumo de álcool.

A não ser nos casos em que o alcoolismo (mais propriamente síndrome de dependência do álcool) é por demais óbvio, ou que exista a possibilidade de uma anamnese muito detaIhada, as informações sobre o consumo de álcool tornam-se, pela sua imprecisão, praticamente inúteis (Masur et al., 1985).

O diagnóstico do alcoolismo é em geral impreciso, freqüentemente subestimado e feito, via de regra, quando o paciente já está num estágio avançado de dependência, com claras repercussões físicas, psíquicas e sociais.
Como contribuição adicional à compreensão maior do alcoolismo, Negrete (1979), nos EUA, relata que a proporção maior deste problema encontra-se entre solteiros, separados e divorciados, sendo portanto o oposto do observado na América Latina. O autor atribui essa diferença a fatos como o predomínio da religião católica nos países latinos e a fatores sócio-econômicos e culturais, os quais levariam a uma maior tolerância entre os casais, evitando separações.

Em virtude da importância desse tema, propusemo-nos a realizar investigações mais sistemáticas, a nível regional, sobre o consumo abusivo do álcool, particularmente os casos que são atendidos em serviços de assistência breve, como setor de emergências.

O objetivo deste trabal ho é apresentar os resultados obtidos de um levantamento de quadros relacionados ao álcool (CID 9 - 291, 303 e 305.0), na região de Ribeirão Preto nos anos de 1988 a 1990, a partir dos atendimentos feitos no único Setor de Urgências Psiquiátricas da Região, inserido num Hospital Escola.

\section{Metodologia}

Esta investigação é um estudo retrospectivo incluindo os casos atendidos nos anos de 1988 a 1990 no Setor de Urgências Psiquiátricas (UE), referido, compreendendo o período de janeiro a dezembro, quando foram considerados todos os pacientes atendidos no setor em horário ininterrupto. A amostra estudada inclui a totalidade de pacientes com distúrbios relacionados ao uso de álcool. Dessa população, foram excluídos os retornos de um mesmo indivíduo. Para os casos em que se verificou a presença de mais de um diagnóstico, usou-se como critério considerar apenas o último.

As informações foram obtidas através do setor de processamento de dados e estatísticas do hospital, ao qual se solicitou um registro do total de pacientes atendidos em cada ano. De posse do material, fez-se uso de um software de informática (Fox-Dbase), que possibilitou o recadastramento, permitindo o agrupamento, auxiliando na análise.

Os diagnósticos foram agrupados conforme - CID 9 (Código Internacional de Doenças), especificando os quadros gerais e também seus subtipos; quanto à procedência, o interesse está central izado nos locais que pertencem à região de Ribeirão Preto - SP, conforme o mapa demográfico de 1989. 
Resultados: análise e discussão

Agrupando-se os dados sobre a população estudada segundo o diagnóstico, na Tabela 1, os principais diagnósticos psiquiátricos atendidos no período estão distribuídos em ordem decrescente. Nessa tabela pode ser observada a freqüência dos distúrbios psiquiátricos na população. Quanto aos diagnósticos de menor relevância numérica, estes foram agrupados sob a denominação "Outros".

A população total atendida no período constou de 5.453 pacientes e o diagnóstico predominante foi o 300 , com um total de 1.707 casos, podendo-se atentar para um aumento significativo deste do ano de 1988 para 1989, mantendo-se elevado nos outros anos. A seguir vem o 295, que apresentou um decréscimo de atendimento de um ano para outro, havendo um pequeno acréscimo no último ano. Os diagnósticos 298, 303, 291, 309, 296, 305.0, 311, 306 e Outros apresentaram aumentos crescentes ano a ano, o que não aconteceu com o diagnóstico 290-294, pois em 1988 apresentou 40 atendimentos, crescendo para 47 em 1989, mas decrescendo para 43 casos em 1990.

Quanto aos diagnósticos relacionados ao consumo de álcool, o que apresentou maior número de casos atendidos foi o 303 (Síndrome de Dependência Alcoólica), com um total de 576 atendimentos, tendo um aumento cres-

Tabela 1

Distribuição dos pacientes segundo principais diagnósticos no Setor de Urgências Psiquiátricas do Hospital de Emergência - FMRP-USP. Período 1988 a 1990.

\begin{tabular}{|c|c|c|c|c|}
\hline Diagnóstico & 1988 & 1989 & 1990 & Total \\
\hline (300) Transtorno N eurótico & 469 & 618 & 620 & 1.707 \\
\hline (295) Psicose Esquizofrênica & 268 & 252 & 254 & 774 \\
\hline $\begin{array}{l}\text { (298) O utras Psicoses } \\
\text { não O rgânicas }\end{array}$ & 140 & 215 & 285 & 640 \\
\hline $\begin{array}{l}\text { (303) Síndrome de } \\
\text { Dependência Alcoólica }\end{array}$ & 153 & 187 & 236 & 576 \\
\hline (291) Psicose Alcoólica & 91 & 108 & 180 & 379 \\
\hline (309) Reações de Ajustamento & 76 & 101 & 115 & 292 \\
\hline (206) Psicoses Afetivas & 28 & 58 & 89 & 175 \\
\hline $\begin{array}{l}\text { (290-294) O utras Psicoses } \\
\text { O rgânicas }\end{array}$ & 40 & 47 & 43 & 130 \\
\hline (305.0) Álcool & 9 & 36 & 82 & 127 \\
\hline $\begin{array}{l}\text { (311) Transtornos Depressivos } \\
\text { não Classificados }\end{array}$ & 28 & 35 & 38 & 101 \\
\hline (306) Disfunção Fisiológica & 34 & 24 & 29 & 87 \\
\hline (O utros)* & 121 & 144 & 200 & 465 \\
\hline Total por ano & 1.457 & 1.825 & 2.171 & 5.453 \\
\hline
\end{tabular}

* Estão inclusos os diagnósticos com menor número de atendimentos. cente ano a ano. A seguir vem o diagnóstico 291 (Psicose Alcoólica), com um total de 379 casos, podendo-se observar que, de 91 casos em 1988, aumentou para 108 em 1989,e em 1990 passou para 180; ou seja, nesse último ano apresentou o dobro de atendimentos de 1988. O terceiro diagnóstico foi o 305.0 (Álcool sem Dependência); este também apresentou aumento crescente: em 1988 houve nove casos, passando para 36 em 1989 e aumentando para 82 em 1990; observa-se que este diagnóstico apresentou uma elevação de cerca de nove vezes de 1988 a 1990. Esse dado permite inferir o aumento da ingestão de bebidas alcoólicas na população.

Somando-se os diagnósticos relacionados ao álcool, tem-se um total de 1.082 casos; sendo assim, esse grupo passa a ocupar o segundo lugar como principal diagnóstico, ou seja, $20 \%$ de todos os atendimentos são referentes ao consumo abusivo de álcool, conforme pode-se visualizar na Figura 1.

Através dessa figura, observa-se o decréscimo de atendimentos referentes aos outros diagnósticos não relacionados ao álcool, ou seja, de $82,6 \%$ de casos atendidos em 1988 , houve um decréscimo para 81,9\% em 1989 e, em 1990, a tendência de queda se mantém, atingindo $77 \%$ de todos os atendimentos. Já os diagnósticos referentes ao consumo de álcool, quando somados, têm um total de $17,4 \%$ de casos atendidos em 1988, sendo que, em 1989, aumentou significativamente para $18,1 \%$ e, em 1990, elevou-se consideravel mente para $23 \%$. Verificase, assim, que o decréscimo de atendimentos dos outros diagnósticos acontece em conseqüência do aumento dos relacionados ao consumo de álcool.

Na Figura 2, pode-se observar a distribuição dos diagnósticos relacionados ao álcool e constata-se que o diagnóstico 303 foi o que apresentou maior número de casos, com 53\%, sendo seguido pelo 295 , com $35 \%$ e pelo 305.0 , com $12 \%$ dos atendimentos no período.

Na Tabela 2, em relação às faixas etárias dominantes, pode-se observar o predomínio das faixas de 25-34 anos, com um total de 370 casos atendidos no período estudado, seguido da faixa entre 35-44 anos, com 364 casos e a faixa de 45-54 anos, com 188 atendimentos. As faixas de 15-24 anos e 55- 64 anos também apresentaram números relevantes que são 89 e 56 respectivamente. As faixas que apresentaram menor número de atendimentos foram as de $<15$, com seis casos, e de $>65$, com nove atendimentos.

Nota-se que, na maioria das faixas (exceto 55-64 anos), o consumo vem crescendo ano a 
ano, para ambos os sexos, embora tal aumento seja mais evidente no sexo masculino.

A maior freqüência de casos de alcoolismo encontra-se nas faixas etárias de 25 a 44 anos, coincidindo, assim, com os dados encontrados no trabalho de Cardin (1985).

Visualiza-se, no total dos atendimentos, o predomínio do sexo masculino. Vale ressaltar que o número de atendimentos no sexo feminino apresenta um aumento uniforme, principalmente nas faixas etárias de 15-24, 35-44 e 45-54 anos, sendo que, na faixa de 25-34, houve um crescimento instável, pois no ano de 1988 teve 11 atendimentos, em 1989 passou para 22 e em 1990 decresceu para 18. Na faixa etária < 15 anos, o número de atendimentos foi igual para os dois sexos, com um número total de seis casos, e, na faixa $>65$, todos os casos atendidos foram do sexo masculino. Esse aumento crescente no sexo feminino torna-se preocupante, sendo o mesmo observado nos trabalhos de Hochgraf et al. (1990) e de Monteiro et al. (1991).

\section{Considerações finais}

Através da realização desse estudo, pode-se concluir a importância do alcoolismo como um problema de saúde pública.

Da população total atendida, no período estudado, somando-se os diagnósticos referentes ao consumo de álcool, estes ficam em segundo lugar na relação de diagnósticos principais, sobressaindo-se do número referente às Psicoses Esquizofrênicas (295). Cardin (1986), comparando trabalhos sobre alcoolismo, segundo diversos autores, ressaltou que a esquizofrenia e alcoolismo somados chegariam a alcançar quase $50 \%$ dos diagnósticos psiquiátricos e, efetuando-se uma média, o alcoolismo encontrava-se como quarto diagnóstico psiquiátrico. Salienta-se que a maior parte dos estudos foram real izados entre as décadas de 30 e 50, mas na década de 70, Madalena (1980) e Viana Filho (1974-1975) encontraram o alcoolismo como segundo diagnóstico psiquiátrico, sendo superado apenas pela Psicose Esquizofrênica.

Esses dados são referentes às internações, pois não foi encontrado esse tipo de estudo em atendimentos de urgências psiquiátricas; portanto, não se encontraram, na literatura, trabaIhos que permitissem comparações sobre os achados.

Neste trabalho, os casos referentes ao consumo de álcool constituíram $19,9 \%$ de todos os casos atendidos, tendo sido observado um au-
Figura 1

Comparação dos números de atendimentos entre os diagnósticos relacionados ao consumo de álcool e demais diagnósticos. Período 1988-1990.

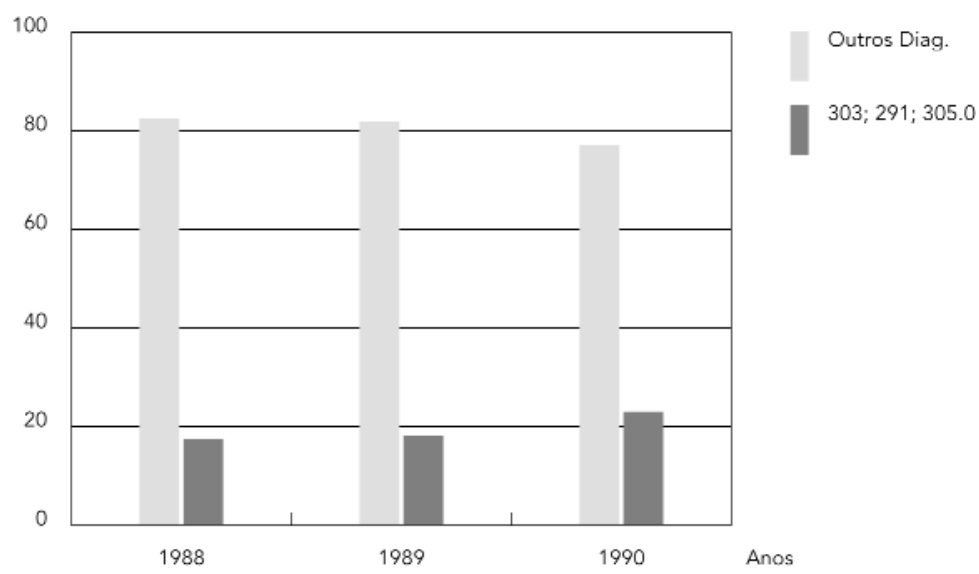

Figura 2

Distribuição dos diagnósticos relacionados ao consumo de álcool, atendidos no Setor de Urgências Psiquiátricas da Região de Ribeirão Preto, SP. Período 1988-1990.

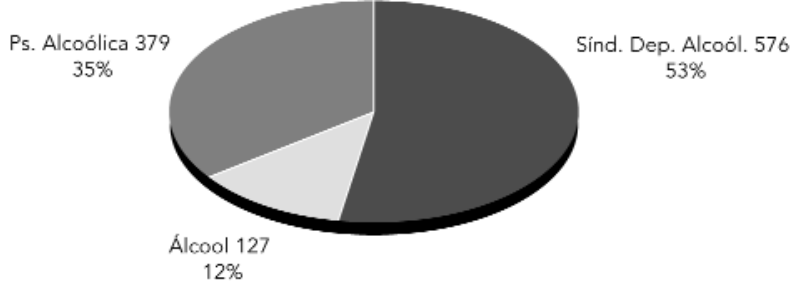

Tabela 2

Distribuição dos pacientes atendidos com diagnósticos relacionados ao consumo de álcool, por sexo e faixa etária. Período 1988-1990.

\begin{tabular}{lrrrrrrrrr}
\hline \multirow{2}{*}{$303,291,305.0$} & \multicolumn{1}{c}{1988} & \multicolumn{2}{c}{1989} & \multicolumn{2}{c}{1990} & \multicolumn{2}{c}{ Total } & Total geral \\
& M & $F$ & M & $F$ & M & $F$ & M & $F$ & \\
\hline$<15$ & - & 2 & 1 & - & 2 & 1 & 3 & 3 & 6 \\
$15-24$ & 19 & 2 & 18 & 3 & 41 & 6 & 78 & 11 & 89 \\
$25-34$ & 73 & 11 & 93 & 22 & 153 & 18 & 319 & 51 & 370 \\
$35-44$ & 75 & 9 & 97 & 17 & 146 & 20 & 318 & 46 & 364 \\
$45-54$ & 43 & 5 & 50 & 8 & 68 & 14 & 161 & 27 & 188 \\
$55-64$ & 10 & - & 18 & 2 & 21 & 5 & 49 & 7 & 56 \\
$>65$ & 4 & - & 2 & - & 3 & - & 9 & - & 9 \\
& & & & & & & & & \\
Total por ano & 224 & 29 & 279 & 52 & 434 & 64 & 973 & 145 & 1.082 \\
\hline
\end{tabular}


mento crescente ano a ano. Os dados confirmam o alcoolismo como um problema médico-social na região, pois é sabido que esses pacientes somente procuram o serviço em período de crises, não retornando senão para novas internações.

O sexo predominante foi o masculino, em todos os anos, e o sexo feminino apresentou números relativamente baixos se comparados ao primeiro, mas salienta-se a importância do número crescente de atendimentos entre as muIheres, principalmente, no concernente ao diagnóstico 303 (Síndrome de Dependência Alcoólica) e 291 (Psicose Alcoólica). Cardin et al. (1986), através de levantamento de literatura, detectaram esse aumento progressivo de alcoolismo na mulher, na média de 10 homens para 1 mulher, e tentaram explicá-lo através das más condições de vida que obrigam a muIher a trabalhar desde cedo, em decorrência de viver em lares desintegrados; da inserção da mulher num mercado de trabalho competitivo; da influência da própria sociedade de consumo, que também visa à oferta de produtos específicos às mulheres, como cigarros e bebi- das; e, por último, a solidão e o stress dos grandes centros urbanos, pelos quais passam igualmente o homem e a mulher.

A faixa etária predominante da população atendida foi a de 25-34 e 35-44 anos, sendo esse o grupo da população economicamente mais ativo. A faixa de 45-54 anos também apresentou número relevante de casos. Tais dados coincidem com os encontrados por Cardin et al. (1986), que assinalam a predominância do abuso de álcool em adultos jovens entre 20-49 anos de idade.

Observou-se, através desse estudo, um aumento crescente tanto no que se refere aos tipos de diagnósticos relacionados ao álcool, como no que se refere à população mais atingida em termos de sexo e faixa etária dos usuários, tornando-se relevante para a detecção do uso abusivo de álcool, numa amostra da população freqüentadora de serviço de atendimento breve (Urgência Psiquiátrica). Espera-se que os resultados obtidos sejam incentivo para os profissionais da área realizarem estudos semeIhantes em seus locais de origem, uma vez que o conhecimento pode facilitar a intervenção.

\section{Referências}

APA (American Psychiatric Association), 1987. Diagnostic and Statistical Manual of Disorders. 3rd ed., Washington, DC: APA.

AZOUBEL-NETO, D., 1965. Contribuição para o Estudo Epidemiológico do Al coolismo. Tese de Doutorado. Ribeirão Preto: Faculdade de Medicina, Universidade de São Paulo.

BERTOLOTE, J. M., 1979. Epidemiologia do alcoolismo: alternativas metodológicas para seu estudo. Arquivos da Clinica Pinel, 5:176-185.

CABERNITE, L., 1982. O alcoolismo no Brasil e as dificuldades na área - Epidemiologia e Prevenção. Jornal Brasileiro de Psiquiatria, 31:89-112.

CARDIN, M. S.; ASSIS, S. G; SBERZE, M.; IGUHI, T. \& MORGADO, A. F., 1986. Epidemiologia descritiva do alcoolismo em grupos populacionais do Brasil. Cadernos de Saúde Pública, 2:191-211.

CERVESATO, A. C. Z., 1993. Alcoolismo: como conhecer e tratar. Jornal dos Psicólogos da Saúde em Pernambuco, Recife. Ano I, no 2.

EDWARDS, G. O., 1985. O Tratamento do Al coolismo. São Paulo: Editora Martins Fontes.

FORTES, J. R. de A., 1975. Alcoolismo. São Paulo: Savier.

FRIDMAN, I. S. \& PELLEGRINI, I. L., 1994. Trabalho e Drogas: Uso de Substâncias Psi coativas no TrabaIho. Dissertação de Mestrado. Porto Alegre: Pontifícia Universidade Católica do Rio Grande do Sul.

HILL, S., 1985. The disease concept of alcoholism: a review. Drug and Alcohol Dependence, 16:193214.
HOCHCRAF, P. B.; TUCCI, M. R.; ZOBERMAN, M. L. \& ANDRADE, A. G., 1990. Comparação entre muIheres e homens alcoolistas em relação a dados sócio-demográficos e outras características relacionadas ao uso de álcool. Revista ABP-APAL, 12:25-30.

HOLDEN, C., 1987. Alcoholism and the medical cost crunch. Science, 235:1132-1133.

LARANJEIRA, R., 1995. Bases para uma Política de Treinamento dos Problemas Rel acionados ao Álcool e Outras Drogas no Estado de São Paulo. Unidade de Pesquisa em Álcool e Drogas. Escola Paulista de Medicina, Universidade Federal do Estado de São Paulo. (mimeo.)

MADALENA, J. C., 1980. A Epidemiologia das Doenças Mentais. Rio de Janeiro.

MASUR, J. \& DEL PORTO, J. A., 1982. Nonmedical treatment of alcoholism with emetic drugs and dissulfiram in Brasil. Journal of Studies on Alcohol, 42:814-817.

MASUR, J., 1984. A Questão do Alcoolismo. São Paulo: Ed. Brasiliense.

MASUR, J.; CAPRIGLIONE, M. J.; MONTEIRO, M. G. \& JORGE, M. R., 1985. Detecção precoce do alcoolismo em clínica médica através do questionamento CAGE: utilidade e limitações. Jornal Brasileiro de Psiquiatria, 34:31-34.

MASUR, J. \& JORGE, M. R., 1986. Dados relacionados a bebidas alcoólicas e alcoolismo no Brasil: uma revisão. Revista ABP-APAL, 8:157-165. 
MS (Ministério da Saúde), 1988. Programa Nacional de Controle dos Problemas Relacionados com o Consumo de Álcool - PRONAL. Brasília: MS/ Disan.

MONTEIRO, M. G.; MONTEIRO, M. A. \& SANTOS, B. R., 1991. Detecção do alcoolismo na população geral através do questionamento CAGE: o que mudou em cinco anos. Revista ABP-APAL, 13:4547.

NAVEILLAN, P. \&VARGAS, S., 1984. Expectativa de vida del bebedor problema en Santiago, Chile. Boletín dela Organización Panamericana deSalud, 97:252-260.

NAVEILLAN, P. \& VARGAS, V., 1989. Procedencia del alcoholismo durante tres décadas en Chile (19521982). Revista de Saúde Pública, 23:128-135.

NEGRETE, J. C., 1976. El alcohol y las drogas como problemas de salud em América Latina. Bol etín de la Organización Panamericana de Salud, 81:158-169.

OMS (Organización Mundial de la Salud), 1970. Comitéde Expertos dela OMS en Farmacodependencia. Genebra: OMS (Serie de Informes Técnicos 437).

POIKOLAINEN, K., 1987. Alcohol and healt: new perspectives in survey research. In: Psychiatric Epidemiology (B. Cooper, ed.), p. 407, London: Croom Helm.

RAEDER, J. R. \& CARLINI-COTRIN, B., 1990. Internações hospitalares no Brasil por dependência de drogas, álcool e psicoses alcoólicas, em 1988. Revista ABP-APAL, 12:33-39.
REGINATO, W.; M ORAES-FILHO, J. P., 1986. Álcool e alcoolismo. Revista Brasileira de Clínica eTerapêutica, 15:79-82.

REGO, R. A.; OLIVEIRA, Z. M. A; BERALDO, F. A. N.; OLIVEIRA, M. B. \& RAM OS, R. L., 1991. Epidemiologia do alcoolismo. Prevalência de positividade do teste CAGE em inquérito domiciliar no Município de São Paulo. Revista ABP-APAL, 13:75-80.

ROBINS, L. N.; HELZER, J. E. \& WEISSM AN, M. N., 1984. Lifetime prevalence of specific psychiatric disorders in three sites. Archives of General Psychiatry, 41:949-958.

SCHUCKIT, M., 1985. Overview: epidemiology of alcoholism. In: Alcohol Pattens \& Problems (M Schuckit, eds.), p. 274, New Jersey: Rutgers University Press.

SSPESP (Secretaria de Segurança Pública e da Promoção do Estado de São Paulo), 1980. O Al coolismo nos Conflitos Familiares: Uma Abordagem Criminológica. São Paulo: Governo do Estado de São Paulo.

VAILLANT, G. E., 1983. The Natural History of Alcoholism. Massachussets: Havard University Press.

VIANA FILHO, A., 1974-75. Levantamento Epidemiológico de Al coolismo na Il ha de Santa Catarina. Florianópolis: Departamento Autônomo de Saúde Pública, Seção de Saúde Mental. (mimeo.)

WALSH, B. \& GRANT, M., 1985. Internactional trends in alcohol production and consumption: implications for public health. Rapport Trimestrel Statistique Sanitaires Mondiales, 38:130-141. 